\section{Analysis of mineral apposition rates during alveolar bone regeneration over three weeks following transfer of BMP-2/7 gene via in vivo electroporation}

\author{
Mariko Kawai, ${ }^{1,2,3 *}$ Yohei Kataoka, ${ }^{2 *}$ \\ Junya Sonobe, ${ }^{3}$ Hiromitsu Yamamoto, \\ Hiroki Maruyama, ${ }^{4}$ \\ Toshio Yamamoto, ${ }^{2 \S}$ Kazuhisa Bessho, \\ Kiyoshi Ohura ${ }^{1}$ \\ ${ }^{1}$ Department of Pharmacology, Osaka \\ Dental University \\ ${ }^{2}$ Department of Oral Morphology, \\ Okayama University Graduate School of \\ Medicine, Dentistry and Pharmaceutical \\ Sciences, Okayama \\ ${ }^{3}$ Department of Oral and Maxillofacial \\ Surgery, Graduate School of Medicine, \\ Kyoto University \\ ${ }^{4}$ Department of Clinical Nephroscience, \\ Niigata University Graduate School of \\ Medicine and Dental Sciences, Niigata, \\ Japan
}

*These authors contributed equally to this work

${ }^{\S}$ Current status: Professor Emeritus at Okayama University, Okayama, Japan

\section{Abstract}

Alveolar bone is not spontaneously regenerated following trauma or periodontitis. We previously proposed an animal model for new alveolar bone regeneration therapy based on the non-viral $B M P-2 / 7$ gene expression vector and in vivo electroporation, which induced the formation of new alveolar bone over the course of a week. Here, we analysed alveolar bone during a period of three weeks following gene transfer to periodontal tissue. Non-viral plasmid vector pCAGGS-BMP-2/7 or pCAGGS control was injected into palatal periodontal tissue of the first molar of the rat maxilla and immediately electroporated with 32 pulses of $50 \mathrm{~V}$ for $50 \mathrm{msec}$. Over the following three weeks, rats were double bone-stained by calcein and tetracycline every three days and mineral apposition rates (MAR) were measured. Double bonestaining revealed that MAR of alveolar bone was at similar level three days before $B M P-2 / 7$ gene transfer as three days after gene transfer. However, from 3 to 6 days, 6 to 9 days, 9 to 12 days, 12 to 15 days, 15 to 18 days, and 18 to 20 days after, MARs were significantly higher than prior to gene transfer. Our proposed gene therapy for alveolar bone regeneration combining nonviral $B M P-2 / 7$ gene expression vector and in vivo electroporation could increase alveolar bone regeneration potential in the targeted area for up to three weeks.

\section{Introduction}

Alveolar bone plays an important role in supporting dental implants and teeth. ${ }^{1}$ However, it cannot be regenerated spontaneously in the case of damage caused by trauma or periodontitis. ${ }^{2,3}$ Therefore, patients undergoing procedures for dental implants but with reduced alveolar bone, require alveolar bone regeneration treatments such as autotransplantation from the patient's mandibular or iliac bone or transplantation of artificial bone. ${ }^{4-6}$ However, as all these therapies are accompanied by surgical procedures, patients face the risk of complications, such as post-operative infection or fractures. ${ }^{7-9}$ Therefore, effective nonsurgical therapy for alveolar bone regeneration offers an appealing clinical alternative to such patients.

In our previous studies, we induced ectopic bone formation by delivering the bone morphogenetic protein (BMP)-2 gene to skeletal muscles of rats without surgical procedure. ${ }^{10-13}$ Although BMP-2 recombinant protein had strong osteoinductive potential, it required proper and biocompatible carriers to prevent it from diffusing out of the target area. ${ }^{14}$ Indeed, direct application of the recombinant BMP-2 protein for alveolar bone regeneration, required surgical operation to retain the protein's carrier on the targeted periodontal tissue. ${ }^{15,16}$ To avoid such drawbacks, we opted for the BMP gene as the delivery reagent to the target sites. Furthermore, to maximize osteoinduction compared with BMP-2 or BMP-7 homodimers, we constructed a BMP-2/7 heterodimer carried by a non-viral plasmid vector. ${ }^{17,18}$ The $B M P-2 / 7$ gene expression vector could calcify ectopic bone formation more quickly than BMP-2 vector. ${ }^{18}$ We thus proposed $B M P-2 / 7$ gene transfer to periodontal tissue with in vivo electroporation for non-surgical alveolar bone regeneration therapy. ${ }^{19}$ During a week, new alveolar bone was induced in the target periodontal tissues and connected with original alveolar bone without bone resorption, as suggested by hematoxylin and eosin (H\&E) staining of tissue sections. ${ }^{19}$ However, as the alveolar bone was observed only for a week, which is too short a time to confirm its clinical use, here, we sought to analyze alveolar bone after $B M P-2 / 7$ gene transfer for a longer time. Alveolar bone is always remodelling and changing, ${ }^{20}$ which means that the fragmental changes in bone formation seen by
Corresponding author: Dr. Mariko Kawai, Department of Pharmacology, Osaka Dental University, 8-1 Kuzuhahanazono-cho, Hirakata city, Osaka, 573-1121, Japan.

Tel. +81.72.8643280 - Fax: +81.72.8643283. E-mail: kawai-m@cc.osaka-dent.ac.jp

Contributions: MK, conceived of and designed the experiments, performed immunohistological experiments and analyzed the data; YK, HY, performed immunohistochemical analyses; MK, JS, HM, KB, TY, $\mathrm{KO}$, prepared the manuscript.

Conflict of interest: The authors declare no conflict of interest.

Key words: BMP; gene transfer; in vivo electroporation; alveolar bone; histomorphometry.

Received for publication: 25 May 2018.

Accepted for publication: 31 July 2018

This work is licensed under a Creative Commons Attribution-NonCommercial 4.0 International License (CC BY-NC 4.0).

CC Copyright M. Kawai et al., 2018

Licensee PAGEPress, Italy

European Journal of Histochemistry 2018; 62:2947 doi:10.4081/ejh.2018.2947

H\&E staining, are insufficient to trace its dynamics over time. In this study, we observed the alveolar bone for three weeks after $B M P-2 / 7$ gene transfer to periodontal tissue and evaluated its regeneration by time-dependent histomorphometric analyses using double-staining with calcein and tetracycline. ${ }^{20}$

\section{Materials and Methods}

\section{Gene transfer}

Nine-week-old male Wistar rats $(\mathrm{n}=3$ per group) were anesthetized via an intraperitoneal injection of sodium pentobarbital (5 mg/100 $\mathrm{g}$ body weight). Construction of the BMP-2/7 gene expression plasmid (pCAGGS-BMP-2/7) or empty plasmid (pCAGGS) is detailed in our previous study. ${ }^{18,19}$ Briefly, each vector was diluted to $0.5 \mu \mathrm{g} / \mu \mathrm{L}$ in phosphate-buffered saline, and $50 \mu \mathrm{L}$ was injected into the palatal region of periodontal tissue of the first molar in the maxilla using a syringe with a 31-gauge needle. In vivo electroporation was performed immediately with 32 pulses of $50 \mathrm{~V}$ for $50 \mathrm{msec} .{ }^{21}$ All procedures were approved by the Animal Care and Use Committee of Okayama University (approval number oku-2012137) and Animal Research Committee of Osaka 
Dental University (approval number 151001).

\section{Bone double-staining}

Nine-week-old male Wistar rats $(\mathrm{n}=3$ per group) were injected intraperitoneally with calcein $(10 \mathrm{mg} / \mathrm{kg}$ body weight $)$ three days before gene transfer. Three days later, tetracycline hydrochloride $(30 \mathrm{mg} / \mathrm{kg}$ body weight) was injected intraperitoneally and gene transfer was performed. Rats were again injected with calcein on days 3, 9, 15, and 20 after gene transfer; and with tetracycline on days 6,12 , and 18 after gene transfer. They were sacrificed with an overdose of sodium pentobarbital on day 21 after gene transfer (Figure 1). The maxillary regions of rats were dissected and fixed with $70 \%$ ethanol for eight days, stained with Villanueva osteochrome bone stain for ten days, dehydrated with increasing concentrations of ethanol, and embedded in methyl methacrylate without decalcification. ${ }^{22}$ After polymerization, $10-\mu \mathrm{m}$ frontal sections were obtained from the mesiolingual center of the upper first and second molars; the region of alveolar bone surrounding the second molar was used as an untreated control for the experiment. Sections were observed by fluorescence microscopy under UV-visible irradiation to detect tetracycline $(364 \mathrm{~nm})$ and calcein (477 nm) staining. The distance between the calcein and tetracycline signal was measured vertically in ten different points within the region affected by gene transfer using a Histometry RT Camera (System Supply, Tokyo, Japan). The 10 points measured were selected in which signals with calcein and tetracycline were lined horizontally and the surface of alveolar bones were not rough. Statistical analyses were performed using an unpaired two tail's $t$-test.

\section{Results}

\section{Villanueva bone staining}

Villanueva osteochrome bone staining (Figure 2) was used to evaluate alveolar bone changes three weeks after gene transfer in the region of the first molar, and compare them to the control groups. Following this staining, the osteoid appears transparent green to jade green or homogeneous red, low-density bone is red, the nuclei of osteoblasts or osteocytes are greenish-blue to dark purple.

\section{Bone labeling and mineral apposi- tion rate (MAR)}

We found nine sites with double-staining in the alveolar bones of the first and second molars (Figure 3). Lines 8 (correspond- ing to tetracycline) and 9 (corresponding to calcein) exhibited only weak staining because the intervals between subsequent injections were shorter (two days and one day, respectively) compared with those of other lines. We determined MARs by measuring the distance between the calcein and tetracycline signals. The width of the areas labeled with calcein and tetracycline was measured vertically at 10 points. The baseline level was set as the MAR value three days before gene transfer; MAR values for the first molar (Figure $4 \mathrm{~A}, \mathrm{~B}$ ) or second molar (Figure 4 C,D) after gene transfer were then compared to it. These were assessed from 0 to 3 days, 3 to 6 days, 6 to 9 days, 9 to 12 days, 12 to 15 days, 15 to 18 days, and 18 to 20 days. MAR values for the first and second molars in the empty plasmid vector group were not significantly different (Figure 4 A,C). MAR values for the first molar from 3 to 6 days, 6 to 9 days, 9 to 12 days, 12 to 15 days, 15 to 18 days, and 18 to 20 days after gene transfer were significantly different from those before gene transfer (Figure 4B). In comparison, MAR

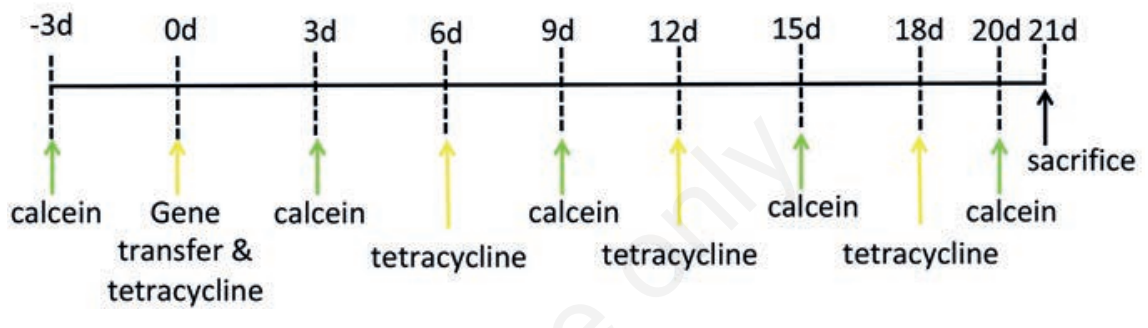

Figure 1. Schematic representation of injections for double bone staining. Rats were first injected intraperitoneally with calcein. Three days later, tetracycline hydrochloride was injected and gene transfer was performed. Rats were again injected with calcein on days $3,9,15$, and 20 after gene transfer; and with tetracycline on days 6,12 , and 18 after gene transfer. They were sacrificed on day 21 after gene transfer.
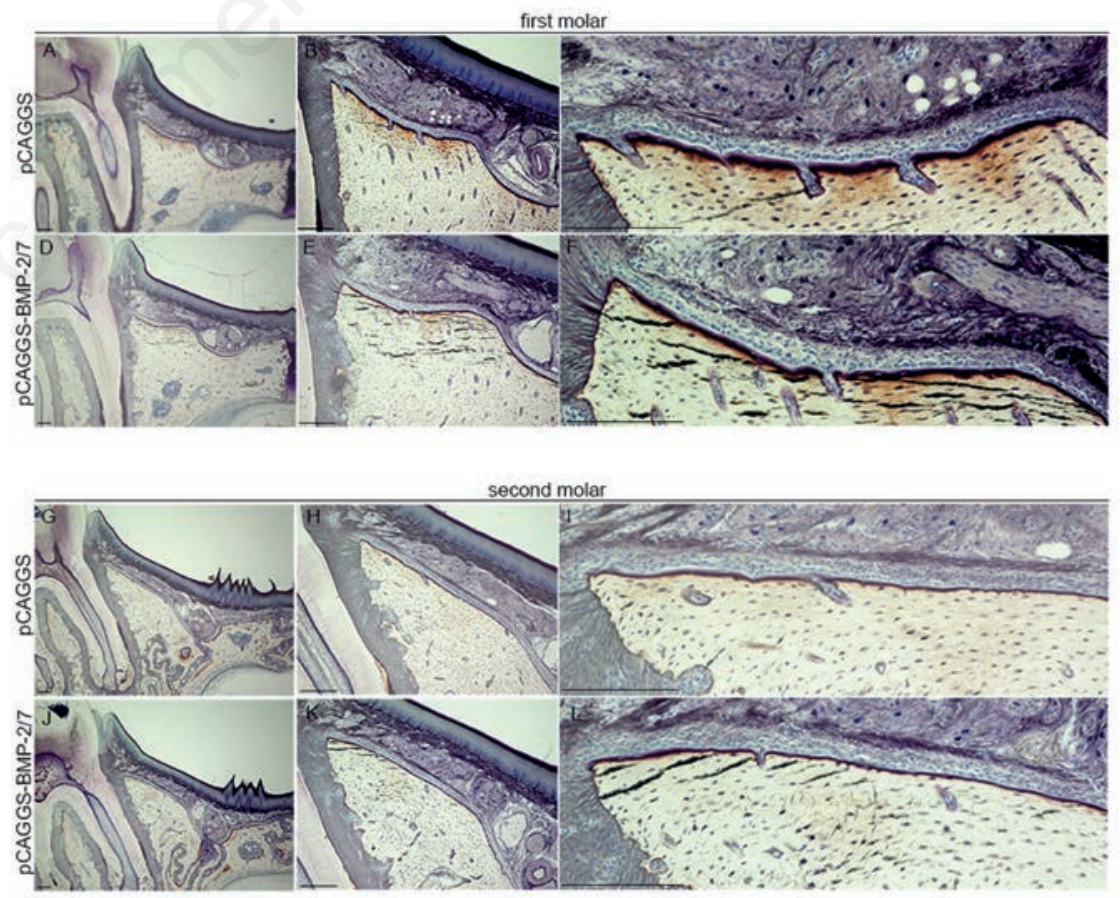

Figure 2. Villanueva bone staining three weeks after gene transfer. Periodontal tissue, including alveolar bone, in the middle of the first molar following electroporation with the empty vector $\mathrm{pCAGGS}(\mathrm{A}, \mathrm{B}, \mathrm{C})$ and $\mathrm{pCAGGS}-\mathrm{BMP}-2 / 7$ vector $(\mathrm{D}, \mathrm{E}, \mathrm{F})$. Periodontal tissue, including alveolar bone, in the middle of the second molar following electroporation of the first molar with the empty vector pCAGGS (G,H,I) pCAGGS-BMP-2/7 vector (J,K,L). Scale bar: $200 \mu \mathrm{m}$. 
values for the second molar did not differ significantly (Figure 4D). Furthermore, we detected significant differences in total mineral apposition rates from 0 to 21 days following gene transfer (Figure 4B). Therefore, $B M P-2 / 7$ gene transfer to periodontal tissues was found to increase mineral apposition rates (Figure 4B).

\section{Discussion}

In this study, we evaluated alveolar bone regeneration for three weeks after $B M P-2 / 7$ gene transfer to periodontal tissue by measuring MAR in bone double-stained with calcein and tetracycline. We revealed that MAR was significantly higher at 3 to 6 days, 6 to 9 days, 9 to 12 days, 12 to 15 days, 15 to 18 days, and 18 to 20 days after $B M P-2 / 7$ gene transfer than before. In contrast, no significant differences in MAR before and three weeks after gene transfer were seen in the control groups. MAR from 0 to 3 days after $B M P-2 / 7$ transfer was not significantly different from basal MAR levels, which is in line with previous results claiming no significant difference in MARs for up to three days after $B M P-2 / 7$ transfer compared with $B M P-2 / 7$ and $L a c Z$ gene transfer. ${ }^{19}$ After $B M P-2 / 7$ gene transfer to periodontal tissue, CD68, a marker of inflammation, was seen to migrate to the targeted periodontal areas, even though no burn or necrosis of the oral mucosa was observed. ${ }^{19}$ As the inflammatory reaction persisted until three days after gene transfer to periodontal tissue, ${ }^{19}$ we assumed that it might affect MAR during this time, thus retarding by three days the effect of exogenous $B M P-2 / 7$ on alveolar bone.

In this study, we set the MAR value before gene transfer in each group as the baseline. Because remodeling activity in the alveolar bone is different for each tooth or part of bone tissue, ${ }^{23}$ we chose to compare MAR values for the same type of tooth from each individual rat to the corresponding baseline value. A comparison of the average MAR value 20 days after gene transfer revealed a significantly higher MAR in the first molar of the $B M P-2 / 7$ gene transfer group than either in the control group or in the second molar (data not shown). However, it should be noted that MAR values for the first and second molars were significantly different even before gene transfer (data not shown). Moreover, standard deviation was smaller for the $B M P-2 / 7$ gene transfer group than for the control group.

In our previous study, we detected exogenous BMP-2 or BMP-7 protein in target periodontal tissue up until three days after $B M P-2 / 7$ gene transfer using immunohistochemistry analysis. ${ }^{19}$ Moreover, new alveolar bone tissue was detected in the target site five days after $B M P-2 / 7$ gene transfer and was connected with original alveolar bone seven days after gene transfer. The area of osteoid was much wider in periodontal tissue following BMP-2/7 gene transfer than in the control site. ${ }^{19}$ However, three weeks after $B M P-2 / 7$ gene transfer, Villanueva osteochrome bone staining did not reveal any dramatic difference compared to the control group. This lack of any visible change could be explained by the fact that in this study, we performed only one session of $B M P-2 / 7$ gene transfer to periodontal tissue. Therefore, it was unexpected for us that a single $B M P-2 / 7$ gene transfer to periodontal tissue was sufficient to affect mineral apposition potential over a period of three weeks. Based on histomorphometric analyses, MAR revealed dynamic changes in bone tissue, ${ }^{24-26}$ complementing the qualitative and static evaluations obtained previously by histological analyses. ${ }^{27}$ Therefore, the present histomorphometric results might offer a more accurate and dynamic representation of alveolar
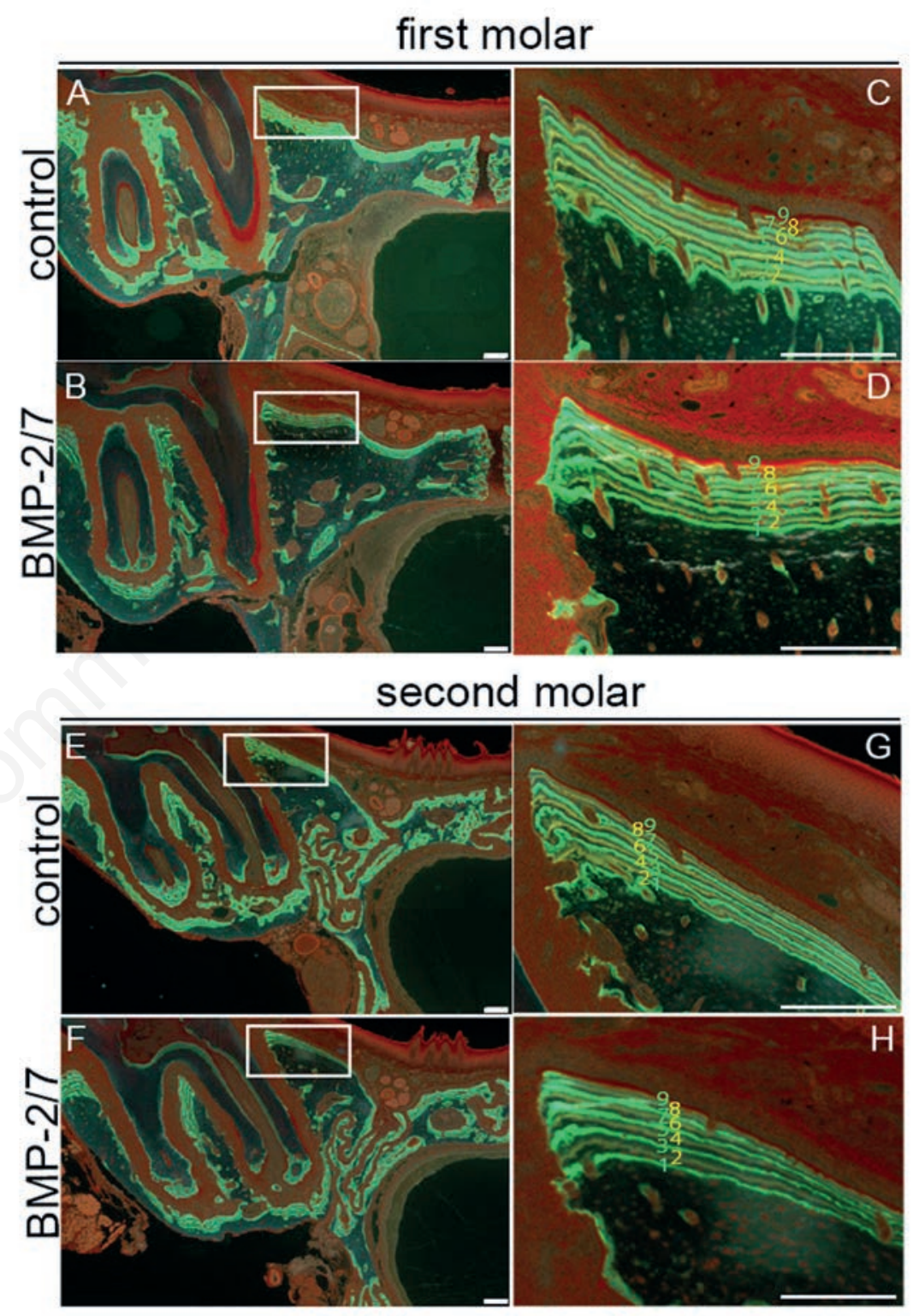

Figure 3. Histomorphometric analysis by double-staining with calcein (green line) and tetracycline (yellow line) in the alveolar bone of the first or second molars. Alveolar bone in the first molar following electroporation with pCAGGS $(\mathrm{A}, \mathrm{C})$. Alveolar bone in the second molar following electroporation of the first molar with pCAGGS (E,G). Alveolar bone in the first molar following electroporation with pCAGGS-BMP-2/7 (B,D). Alveolar bone in the second molar following electroporation of the first molar with pCAGGS-BMP-2/7 (F,H). The nine lines were labeled by calcein and tetracycline (C,D,G, ). Scale bar: $200 \mu \mathrm{m}$. 

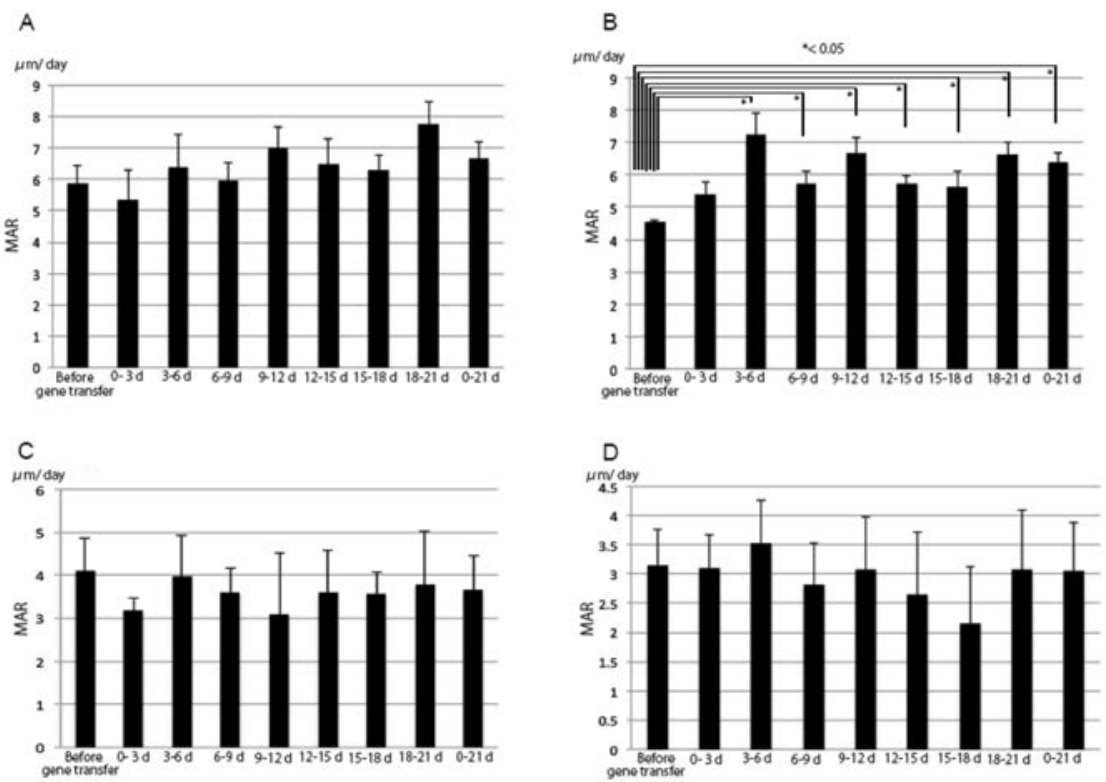

Figure 4. MAR of alveolar bone in the first and second molars following electroporation with pCAGGS or pCAGGS-BMP-2/7. MAR of alveolar bone in the first molar electroporated with pCAGGS (A). MAR of alveolar bone in the first molar electroporated with pCAGGS-BMP-2/7 (B). MAR of alveolar bone in the second molar following transfer of pCAGGS to the first molar (C). MAR of alveolar bone in the second molar following transfer of pCAGGS-BMP-2/7 to the first molar (D). MAR basal values were set based on recordings made 0 to 3 days before gene transfer. The column marked as " $0-21 d$ " displays the average MAR value for the entire period of 0-21 days after gene transfer for each group. Error bars represent the standard deviation of the mean. ${ }^{*} \mathrm{P}<0.05$ for MAR $v$ s basal MAR values resulted by unpaired two tailed $t$-tests.

bone regeneration by $B M P-2 / 7$ gene transfer to periodontal tissue. However, continuously high MARs could sometimes induce bone and mineral disorders such as sclerosis. ${ }^{28,29}$ In the future, alveolar bone regeneration should be followed by histomorphometric analyses for longer than three weeks and a larger rat cohort should be employed to ascertain the method's suitability for clinical use.

In the past, gene therapy has been used to target general disorders, rather than local disease or trauma. ${ }^{30-32}$ However, alveolar bone regeneration therapy is very critical to a restricted regeneration area because alveolar bone develops on a smaller scale and has a smaller structure compared with other bones such as the femur or humerus. ${ }^{33}$ This implies a tight control of the gene delivery area. In our gene transfer system using a non-viral plasmid vector and in vivo electroporation, the electrodes played an important role in restricting the gene transfer area, ${ }^{34}$ as gene transfer could occur only in the area turned on by electricity. ${ }^{34}$ MARs of alveolar bone in the second molar were not significantly different from corresponding basal levels before gene transfer. These results demonstrate that $B M P-2 / 7$ gene transfer to periodontal tissue of the first molar affected only the target site and did not spill over to the second molar. Our present results based on histomorphometric analyses reveal that our previously developed $B M P-2 / 7$ gene transfer system to periodontal tissue could promote alveolar bone regeneration for up to three weeks.

We expect that the proposed method can be applied for clinical use in the form of repeated gene transfer following radiographic examination of the teeth to diagnose the degree of alveolar bone regeneration.

\section{References}

1. Gher ME. Changing concepts. The effects of occlusion on periodontitis. Dent Clin North Am 1998;42:285-99.

2. Fu JH, Yap AU. Occlusion and periodontal disease--where is the link? Singapore Dent J 2007;29:22-33.

3. Tan WL, Wong MC, Lang NP. A systematic review of post-extractional alveolar hard and soft tissue dimensional changes in humans. Clin Oral Implants Res 2012;5:1-21.

4. Peleg M, Sawatari Y, Marx RN, Santoro J, Cohen J, Bejarano P, et al. Use of corticocancellous allogeneic bone blocks for augmentation of alveolar bone defects. Int J Oral Maxillofac Implants 2010;25:153-62.

5. Caubet J, Petsold C, Saez-Torres C, Morey M, iriarte JI, Sánchez J, et al. Sinus graft with safescraper: 5-year results. J Oral Maxillofac Surg 2011;69:482-90.

6. Castagna L, Polido WD, Soares LG, Tinoco EM. Tomographic evaluation of iliac crest bone grafting and the use of immediate temporary implants to the atrophic maxilla. Int J Oral Maxillofac Surg 2013;42:1067-72.

7. de Jong T, Baller AD, EvertsV, Smit $\mathrm{TH}$. The intricate anatomy of the periodontal ligament and its development: lessons for periodontal regeneration. J Periodont Res 2017;52:965-74.

8. Alzarea BK. Assessment and evaluation of quality of life (OHRQoL) of patients with dental implant using the oral health impact profile (OHIP-14)-A clinical study. J Clin Diagn Res 2016;10:57-60.

9. Huynh-Ba G, Friedberg JR, Vogiatzi D, Ioannidou E. Implant failure predictors in the posterior maxilla: a retrospective study of 273 consecutive implants. J Periodontol 2008;79:2256-61.

10. Kawai M, Bessho K, Kaihara S, Sonobe J, Oda K, Iizuka T, et al. Ectopic bone formation by human bone morphogenetic protein-2 gene transfer to skeletal muscle using transcutaneous electroporation. Hum Gene Ther 2003;14: 1547-56.

11. Kawai M, Bessho K, Maruyama H, Miyazaki J, Yamamoto T. Human BMP2 gene transfer using transcutaneous in vivo electroporation induced both intramembranous and endochondral ossification. Anat Rec Part A 2005; 287:1264-71.

12. Kawai M, Bessho K, Maruyama H, Miyazaki J, Yamamoto T. Simultaneous gene transfer of bone morphogenetic protein (BMP)-2 and BMP-7 by in vivo electroporation induces rapid bone formation and BMP-4 expression. BMC Musculoskelet Disord 2006;7:1-11.

13. Kawai M, Ohmori Y, Nishino M, Yoshida M, Tabata T, Hirota DS, et al. Determination of cell fate in skeletal muscle following BMP gene transfer by in vivo electroporation. Eur J Histochem 2017;61:2772.

14. Bessho K, Cames DL, Cavin R, Ong JL. Experimental studies on bone induction using low-molecular-weight poly (DLlactide-co-glycolide) as a carrier for recombinant human bone morphogenetic protein-2. J Biomed Mater Res 2002;61:61-5.

15. Cillo JE Jr, Basi D, Peacock Z, Aghaloo $\mathrm{T}$, Bouloux $\mathrm{G}$, Dodson $\mathrm{T}$, et al. 
Proceeding of the American Association of Oral and Maxillofacial Surgeons 2015 Research Summit. J Oral Maxillofac Surg 2016;74:429-37.

16. Tannoury CA, An HS. Complications with the use of bone morphogenetic protein 2(BMP-2) in spine surgery. Spine 2014;14:552-9.

17. Israel DI, Nove J, Kerns KM, KaufmanRJ, RosenV, Cox JM, et al. Heterodimeric bone morphogenetic proteins show wnhanced activities in vitro and in vivo. Growth Factors 1996;13:291-300.

18. Kawai M, Maruyama H, Bessho K, Yamamoto H, Miyazaki J, Yamamoto T. Simple strategy for bone regeneration with a BMP-2/7 gene expression cassette vector. Biochem Biophys Res Commun 2009;390:1012-7.

19. Kawai M, Kataoka YH, Sonobe J, Yamamoto H, Inubushi M, Ishimoto T, et al. Non-surgical model for alveolar bone regeneration by bone morphogenetic protein-2/7 gene therapy. J Periodontol 2017;18:1-18.

20. Kawai M, Ohura K. Applicability of histomorphomery analysis for evaluating alveolar bone regeneration after gene transfer. J Histol Histopathol Res 2017;1:21-2.

21. Yamamoto H, Kawai M, Shiotsu N, Watanabe M, Yoshida Y, Suzuki K, et al. BMP-2 gene transfer under various conditions with in vivo electroporation and bone formation. J Oral Maxillofac Surg Med Pathol 2012;24:49-53.

22. Chanpattane W, Chakrabhand ML. Factors influencing treatment frequency of continuation ECT in schizophrenia. J ECT 2001;17:190-4.

23. Kobayashi Y, Udagawa N. Mechanisms of alveolar bone remodeling. Clin Calcium 2007;17:209-16.

24. Frost M. Tetracycline-based histologicalanalysis of bone remodeling. Calcif Tissue Res 1969;3:211-37.

25. Parfitt AM. The cellular basis of bone remodeling: the quantum concept reexamined in light of recent advances in the cell biology of bone. Calcif Tissue Int 1984;36:37-45.

26. Mashiba T. Morphological analysis of bone dynamics and metabolic bone disease. Histological findings in animal fracture model-effests of osteoporosis treatment drugs on fracture healing process. Clin Calcium 2011;21:551-8.

27. Yamamoto N, Shimakura T, Takahashi H. Bone cell biology assessment by microscopic approach. Bone histomorphometry of remodelling, modelling and minimodeling. Clin Calcium 2015;25:1491-7.

28. Neves CL, dos Reis LM, Batista DG, Custodio MR, Graciolli FG, de Cassia
TR, et al. Persistence of bone and mineral disorders 2 yeas after successful kidney transplantation. Transplantation 2013;96:290-6.

29. Susan M, Ott MD. Bone histomorphometry in renal osteodystrophy. Semin Nephrol 2009;29:122-32.

30. Dobayashi M, Goda K, Maruyama H, Fujisawa M. Erythropoietin gene transfer into rat testes by in vivo electroporation may reduce the risk of germ cell loss caused by cryptorchidism. Asian J Androl 2005;7:369-73.

31. Abe S, Hanawa H, Hayashi M, Yoshida T, Komura S, et al. Prevention of experimental autoimmune myocarditis by hydrodynamics-based naked plasmid DNA encoding CTLA4-lg gene delivery. J Card Fall 2005;11:557-64.

32. Ataka K, Maruyama H, Neichi T, Miyazaki J, Gejyo F. Effects of erythropoietin -gene electrotransfer in rats with adenine-induced renal failure. Am J Nephrol 2003;23:315-23.

33. Tomokins KA. The osteoimmunology of alveolar bone loss. Connect Tissue Res 2016;57:69-90.

34. Kawai M, Ohura K. Histomorphometric analysis of the alveolar bone for two weeks after bone morphogenetic protein transfer. J Cytol Histol 2017;8:486. 